Check for updates

Cite this: Chem. Sci., 2019, 10, 8338

๑ All publication charges for this article have been paid for by the Royal Society of Chemistry

Received 3rd May 2019

Accepted 28th July 2019

DOI: $10.1039 / \mathrm{c} 9 \mathrm{sc0} 02166 \mathrm{c}$

rsc.li/chemical-science

\title{
Engineering multilayer chemical networks $\dagger$
}

\author{
Maitena Martinez-Amezaga, A. Gastón Orrillo and Ricardo L. E. Furlan (D)*
}

Dynamic multilevel systems emerged in the last few years as new platforms to study thermodynamic systems. In this work, unprecedented fully communicated three-level systems are studied. First, different conditions were screened to selectively activate thiol/dithioacetal, thiol/thioester, and thiol/disulfide exchanges, individually or in pairs. Some of those conditions were applied, sequentially, to build multilayer dynamic systems wherein information, in the form of relative amounts of building blocks, can be directionally transmitted between different exchange pools. As far as we know, this is the first report of one synthetic dynamic chemical system where relationships between layers can be changed through network operations.

\section{Introduction}

The implementation of stable covalent bonds that can be broken and re-formed under certain conditions has paved the way for the construction of robust adaptive systems. ${ }^{1,2}$ Dynamic covalent chemistry has become a valuable tool for different research fields such as materials science, ${ }^{3-7}$ drug discovery ${ }^{8-10}$ and delivery, ${ }^{11,12}$ sensing, ${ }^{13,14}$ and molecular machines. ${ }^{15-17}$

When dynamic covalent chemistry is applied to the preparation of dynamic combinatorial libraries, ${ }^{\mathbf{1 8 , 1 9}}$ library members are interconnected by reversible reactions and can be represented as a network with interlinked nodes, i.e. a monolayer network (Fig. 1A). ${ }^{\mathbf{2 0 2 1}}$ The preparation of complex reaction networks constitutes an important part of systems chemistry, a growing discipline that aims to develop molecular systems of interacting and/or interconverting molecules that show emergent properties. ${ }^{18,22,23}$

In this context, the reversible chemistry used to interconvert library members defines the network wiring and, consequently, affects the system adaptability towards environmental changes. $^{24}$ One way to exert control on network wiring is through the use of more than one reversible reaction within the same system. When two or more reversible reactions are used in one dynamic system, the relative reactivity of the functional groups involved in each reaction will determine many of the system properties. Most of the multi-reaction dynamic systems reported to date were based on different combinations of two orthogonal reactions, ${ }^{25}$ and only a handful involved three or

Farmacognosia, Facultad de Ciencias Bioquímicas y Farmacéuticas, Universidad Nacional de Rosario - CONICET, Suipacha 531, Rosario, S2002SLRK, Argentina. E-mail: rfurlan@fbioyf.unr.edu.ar

$\dagger$ Electronic supplementary information (ESI) available: Synthesis of thioester B1, preparation of one-level, two-level and three-level dynamic chemical systems, full kinetics of isolated-networked experiments and numerical simulations for expected equilibrium compositions when the activation sequences $I-V$ are displayed. See DOI: 10.1039/c9sc02166c more reactions. ${ }^{26-30}$ The one pot combination of reversible exchange chemistries that can intercommunicate ${ }^{31,32}$ has been far less explored than their orthogonal counterpart. ${ }^{33}$ Such a combination generates exchange pools that share one building block type and, consequently, can affect each other. This mutual influence between pools is due to their competence for the building block type they have in common. Control over the composition of such systems has been achieved by controlling the concentration of shared building blocks through irreversible reactions, such as oxidation or reduction, ${ }^{34}$ or changing the reactivity of those shared building blocks

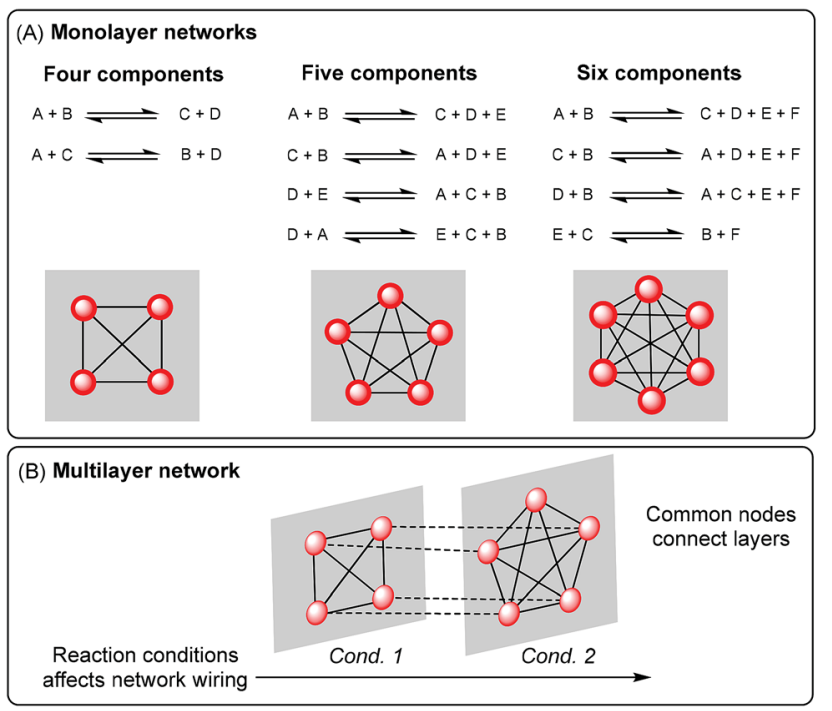

Fig. 1 (A) A fully reversible chemical process involving four, five, or six components described as a set of reactions and as a "monolayer" molecular network. (B) A multilayer network is formed from networks that (i) have at least one node in common and (ii) can be sequentially wired under diverse conditions. 
through isomerization. ${ }^{35}$ In this way, the building blocks can be selectively channelled into different exchange pools.

The combination of communicating reactions that can be sequentially activated represents a special study case since they give rise to multilayer networks. ${ }^{36}$ In multilayer networks, each molecular network is described as a "layer" and the different layers are connected through common nodes, i.e. connector molecules involved in more than one reaction (Fig. 1B). Such a connectivity could mediate the transference of chemical information between different layers because changes in the molecular composition occurring in one layer (that affect the concentration of connector molecules) will be translated to the following layer. So in principle, an imprint of some events occurring along the process could be recorded in the composition and affect ulterior equilibria. ${ }^{20,28}$

The only example of a two-layer dynamic chemical network reported is based on the sequential activation of dithioacetal and disulfide exchanges. ${ }^{36}$ Addition of a third communicating reaction, such as thioester exchange, and the implementation of different reaction conditions to selectively activate the reactions, individually or in pairs, may open the door towards further exploration of multilayer systems.

Here we searched for reagents and conditions to combine three communicating reactions: thiol/disulfide, thiol/thioester, and thiol/dithioacetal exchanges (Fig. 2). Then some selected conditions were applied to build multilayer chemical networks. Although the studied dynamic systems are very simple, they showed some unique properties such as history-dependent information transfer between different compound pools.

\section{Results and discussion}

Initially, a catalyst screening was carried out by mixing dithioacetal 1A1, disulfide 11, thioester B1, and thiol 2H (Fig. 3). These compounds can participate in thiol/dithioacetal exchange, thiol/disulfide exchange or thiol/thioester exchange, three communicating reversible reactions that could in principle be simultaneously or sequentially activated.

Compounds $\mathbf{1 A 1}(30 \mathrm{mM}), \mathbf{1 1}(30 \mathrm{mM}), \mathbf{B 1}(30 \mathrm{mM})$ and $\mathbf{2 H}$ $(60 \mathrm{mM})$ were mixed in acetonitrile at room temperature in the

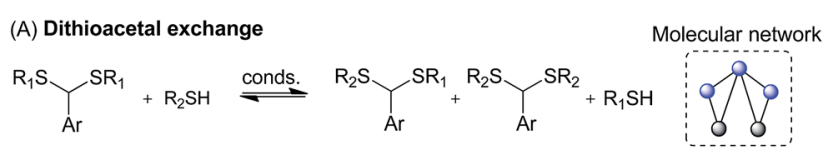

(B) Thioester exchange

$$
\mathrm{R}_{\mathrm{SR}_{1}}^{\mathrm{O}}+\mathrm{R}_{2} \mathrm{SH} \stackrel{\text { conds. }}{\rightleftharpoons} \stackrel{\mathrm{O}}{\stackrel{\mathrm{I}_{\mathrm{SR}_{2}}}{\rightleftharpoons}}+\mathrm{R}_{1} \mathrm{SH}
$$

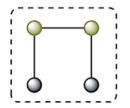

(C) Disulfide exchange

$$
\mathrm{R}_{1} \mathrm{~S}-\mathrm{SR}_{1}+\mathrm{R}_{2} \mathrm{SH} \stackrel{\text { conds. }}{\rightleftharpoons} \mathrm{R}_{1} \mathrm{~S}-\mathrm{SR}_{2}+\mathrm{R}_{2} \mathrm{~S}-\mathrm{SR}_{2}+\mathrm{R}_{1} \mathrm{SH}
$$

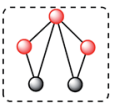

Fig. 2 Reversible reactions used in this study. Dashed line squares show molecular networks of each reaction. Color code for nodes: blue, dithioacetal; black, thiol; olive green, thioester; red, disulfide.
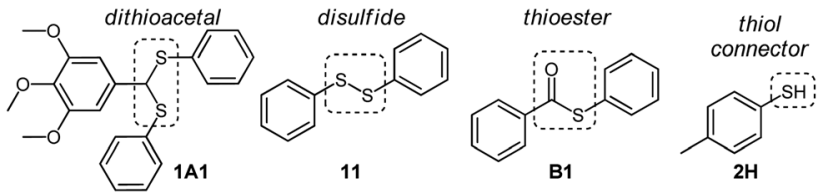

Fig. 3 Building blocks used in this study. Dashed line squares highlight functional groups involved in reversible reactions.

absence or the presence of a catalyst $(30 \mathrm{mM})$, and the composition of the reaction mixture was analyzed after $24 \mathrm{~h}$ by HPLC-UV.

In the absence of any catalyst, thioester $\mathbf{B} 1$ and dithioacetal $1 \mathrm{A1}$ did not react with thiol $\mathbf{2 H}$, whereas disulfide 11 gave rise to an equilibrated mixture of $\mathbf{1 1}, \mathbf{1 2}$ and 22 (Table 1, entry 1).

It is known that Brønsted acids can stop thioester ${ }^{28,37}$ and disulfide $^{38,39}$ exchanges and, at the same time, catalyze dithioacetal exchange. ${ }^{40,41}$ In the presence of trifluoroacetic acid (TFA), disulfides did not exchange; however, the acid concentration $(30 \mathrm{mM})$ was not enough to activate dithioacetal exchange, so the whole system stayed static (entry 2).

At the same concentration, stronger Brønsted acids such as $p$-toluenesulfonic acid (PTSA) or sulfuric acid selectively activated dithioacetal exchange to produce equilibrated mixtures of 1A1, 1A2 and 2A2 (entries 3 and 4).

Several Lewis acids have been reported as dithioacetal formation ${ }^{42-46}$ or hydrolysis catalysts. ${ }^{47,48}$ In our model system, the addition of ytterbium(III) triflate or aluminium(III) chloride

Table 1 Search for conditions for the activation of different combinations of reactions involving dithioacetal, thioester, and disulfide

\begin{tabular}{|c|c|c|c|c|}
\hline Entry & Catalyst & Dithioacetal $^{b}$ & Thioester $^{b}$ & Disulfide $^{b}$ \\
\hline 1 & None & N.R. & N.R. & Equilibrated \\
\hline 2 & TFA & N.R. & N.R. & N.R. \\
\hline 3 & PTSA & Equilibrated & N.R. & N.R. \\
\hline 4 & $\mathrm{H}_{2} \mathrm{SO}_{4}$ & Equilibrated & N.R. & N.R. \\
\hline 5 & $\mathrm{Yb}(\mathrm{OTf})_{3}$ & N.R. & N.R. & N.R. \\
\hline 6 & $\mathrm{AlCl}_{3}$ & N.R. & N.R. & N.R. \\
\hline 7 & $\mathrm{FeCl}_{3}{ }^{c}$ & Equilibrated & N.R. & N.R. \\
\hline 8 & $\mathrm{Cu}(\mathrm{OTf})_{2}{ }^{d}$ & Equilibrated & N.R. & N.R. \\
\hline 9 & $\operatorname{Sn}(\mathrm{OTf})_{2}$ & Equilibrated & N.R. & N.R. \\
\hline 10 & $\mathrm{SnCl}_{2}$ & Slow exchange & N.R. & N.R. \\
\hline 11 & $\mathrm{UV} / \mathrm{DCA}^{c, e}$ & Slow exchange $e^{f}$ & N.R. & Slow exchange \\
\hline 12 & UV/rose bengal ${ }^{g}$ & N.R. & N.R. & Slow exchangef \\
\hline 13 & $\mathrm{TEA}^{c}$ & N.R. & Equilibrated & Equilibrated \\
\hline 14 & DMAP $^{c}$ & N.R. & Equilibrated & Equilibrated \\
\hline 15 & $\left(\mathrm{Bu}_{2} \mathrm{SnCl}\right)_{2} \mathrm{O}^{c}$ & N.R. & N.R. & N.R. \\
\hline 16 & $\left(\mathrm{Bu}_{3} \mathrm{Sn}\right)_{2} \mathrm{O}^{c}$ & N.R. & N.R. & N.R. \\
\hline 17 & $\mathrm{Bu}_{2} \mathrm{SnO}$ & N.R. & N.R. & Equilibrated \\
\hline 18 & $\mathrm{BuSnO}_{2} \mathrm{H}$ & N.R. & Equilibrated & Equilibrated \\
\hline
\end{tabular}
exchanges $^{a}$

${ }^{a}$ Conditions: 1A1 (15 $\left.\mu \mathrm{mol}\right), \mathbf{1 1}(15 \mu \mathrm{mol}), \mathbf{B 1}(15 \mu \mathrm{mol}), 2 \mathbf{H}(30 \mu \mathrm{mol})$, and acetonitrile (total volume: $500 \mu \mathrm{L})$, r.t., and catalyst $(15 \mu \mathrm{mol})$. The compositions were measured by HPLC-UV after $24 \mathrm{~h}$ of reaction $\left(\mathrm{Abs}_{250}\right)$. ${ }^{b}$ N.R.: no product could be detected. ${ }^{c}$ Thiol oxidation was observed. ${ }^{d}$ Decreased thiol concentration. ${ }^{e}$ DCA $(0.15 \mu \mathrm{mol})$ and $\mathrm{O}_{2}$ atmosphere. ${ }^{f}$ Decomposition. ${ }^{g}$ Rose bengal $(0.75 \mu \mathrm{mol})$ and $\mathrm{N}_{2}$ atmosphere. 
did not activate the exchange of dithioacetals or thioesters; besides, it stopped the disulfide exchange (entries 5 and 6). In the presence of iron(III) chloride, only dithioacetal exchange was observed with concomitant thiol oxidation (entry 7). Copper(II) triflate also favored dithioacetal exchange and deactivated disulfide exchange; however, a significant decrease in thiol concentration was observed after $24 \mathrm{~h}$ of reaction (entry 8 ). Two tin(II) reagents were tested: chloride and triflate (entries 9 and 10). Both mild Lewis acids stopped the disulfide exchange and selectively catalyzed dithioacetal exchange; however, the equilibrium was reached in $24 \mathrm{~h}$ only with tin(II) triflate.

The exchange, formation or hydrolysis of dithioacetals has also been achieved using photoirradiation in the presence of photosensitizers such as 9,10-dicyano anthracene (DCA) ${ }^{36}$ or rose bengal. ${ }^{49,50}$

When applied to our starting solution of $\mathbf{1 A 1}, \mathbf{B 1}, \mathbf{1 1}$ and $\mathbf{2 H}$, DCA/UV light induced a very slow exchange of dithioacetals and disulfides along with extensive decomposition (Table 1, entry 11). Similarly, rose bengal/UV light induced disulfide exchange and decomposition, whereas, in this case, dithioacetal and thioester molecules remained unreacted (entry 12).

Thioester exchange in organic solvents is usually achieved with organic bases. ${ }^{37}$ Addition of TEA and DMAP to our model mixture led to the activation of both disulfide and thioester exchanges (entries 13 and 14). Under these basic conditions, thiol oxidation took place and the free thiols could not be detected after $24 \mathrm{~h}$.

Different organotin(Iv) reagents have been used to catalyze carboxylic ester cleavage and/or transesterification; ${ }^{51}$ however, to the best of our knowledge, their use in transthioesterification has not been reported. Therefore, four organotin(Iv) reagents were tested. In the presence of bis(dibutylchlorotin) oxide and bis(tributyltin) oxide the three reactions were inactive (entries 15-16), whereas with dibutyltin oxide only disulfide exchange was active (entry 17). On the other hand, the addition of butylstannonic acid activated thioester and disulfide exchanges, maintaining dithioacetal exchange inactive (entry 18). Despite their differential influence on the thioester exchange, the effect of these reagents on the exchange of disulfides was not expected: two of them inactivated the exchange and the other two did not.

Based on these results, TEA, butylstannonic acid, sulfuric acid, PTSA, tin(II) triflate, and tin(II) chloride were selected for further studies on the isolated reactions as well as binary combinations of simultaneous reactions.

\section{Isolated reactions}

Dithioacetal exchange has been recently introduced into the field of dynamic covalent chemistry. Although so far TFA has been the only Brønsted acid used to catalyze the reaction, ${ }^{36,40,41}$ stronger acids may be more convenient to speed up the exchange (Table 1, entries 3 and 4).

When an acetonitrile solution of dithioacetal 1A1 (30 mM) and thiol $2 \mathbf{H}(60 \mathrm{mM})$ was stirred in the presence of different Brønsted acids (30 $\mathrm{mM})$, the acid strength determined the reaction kinetics. Sulfuric acid equilibrated the mixture within a few minutes, while a tenfold decrease in the amount of acid caused a dramatic increase in the $t_{1 / 2}$ value (Table 2 , entries 1 and 2, see full compositions in Fig. S1 and S2 $\dagger$ ). PTSA led to a $t_{1 /}$ 2 value of $48 \mathrm{~min}$, whereas the same concentration of TFA was not enough to activate the exchange (entries 3 and 4, Fig. S3 and $\mathrm{S} 4 \dagger)$. A ten times higher TFA loading was required to activate the reaction (entry 5, Fig. $\mathrm{S} 5 \dagger$ ).

In addition to Brønsted acids, tin(II) triflate was the only Lewis acid tested that equilibrated dithioacetals without side reactions in a reasonable time frame (Table 2, entries 6-8, Fig. S6-S8†). Tin(II) triflate appears to be a new promising catalyst for dithioacetal exchange.

Previously, Lewis acid catalysis of dithioacetal exchange had only been attempted by Hirsch et al. using $\mathrm{ZnCl}_{2} ;{ }^{52}$ however, the reversibility of the exchange reaction was not confirmed. Interestingly in the DCC context of multilevel systems, tin(II) triflate did not activate thioester exchange and inactivated disulfide exchange.

Transesterification was one of the reactions applied earliest in dynamic combinatorial chemistry. ${ }^{53,54}$ The thioester exchange was introduced later in the discipline, and it has been used to produce several dynamic systems. ${ }^{55-63}$ In organic solvents, this exchange has always been carried out under basic

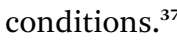

Fast exchange between thioester $\mathbf{B 1}$ and thiol $\mathbf{2 H}$ was catalyzed by stoichiometric or catalytic amounts of TEA (entries 911, Fig. S9-S11†).

In addition to the well-known base-catalyzed thioester exchange, ${ }^{37}$ we studied this reaction with butylstannonic acid (Table 1, entry 18). The reaction with a stoichiometric amount

Table 2 Half-life time for the equilibration of the isolated dithioacetal, thioester or disulfide exchange

\begin{tabular}{|c|c|c|c|c|}
\hline Entry & Exchange reaction & Catalyst & Amount $^{e}$ & $t_{1 / 2}^{f, g}(\min )$ \\
\hline 1 & Dithioacetal $^{a}$ & $\mathrm{H}_{2} \mathrm{SO}_{4}$ & 1 & 1.2 \\
\hline 2 & Dithioacetal $^{a}$ & $\mathrm{H}_{2} \mathrm{SO}_{4}$ & 0.1 & 1800 \\
\hline 3 & Dithioacetal $^{a}$ & PTSA & 1 & 48 \\
\hline 4 & Dithioacetal $^{a}$ & TFA & 1 & N.R. \\
\hline 5 & Dithioacetal $^{a}$ & TFA & 10 & 48 \\
\hline 6 & Dithioacetal $^{a}$ & $\mathrm{Sn}(\mathrm{OTf})_{2}$ & 1 & 8 \\
\hline 7 & Dithioacetal $^{a}$ & $\mathrm{Sn}(\mathrm{OTf})_{2}$ & 0.1 & 8 \\
\hline 8 & Dithioacetal $^{a}$ & $\mathrm{Sn}(\mathrm{OTf})_{2}$ & 0.01 & 800 \\
\hline 9 & Thioester $^{b, c}$ & TEA & 1 & 0.3 \\
\hline 10 & Thioester $^{b, c}$ & TEA & 0.1 & 0.6 \\
\hline 11 & Thioester $^{b, c}$ & TEA & 0.01 & 2.5 \\
\hline 12 & Thioester $^{b}$ & $\mathrm{BuSnO}_{2} \mathrm{H}$ & 1 & 25 \\
\hline 13 & Thioester $^{b}$ & $\mathrm{BuSnO}_{2} \mathrm{H}$ & 0.1 & 420 \\
\hline 14 & Disulfide $^{d}$ & None & - & 17 \\
\hline 15 & Disulfide $^{c, d}$ & TEA & 1 & 0.3 \\
\hline 16 & Disulfide $^{d}$ & $\mathrm{BuSnO}_{2} \mathrm{H}$ & 1 & 0.8 \\
\hline 17 & Disulfide $^{c, d}$ & TFA & 1 & N.R. \\
\hline
\end{tabular}

${ }^{a} 1 \mathrm{A1}(15 \mu \mathrm{mol}), 2 \mathrm{H}(60 \mu \mathrm{mol})$, and acetonitrile $(500 \mu \mathrm{L}) .{ }^{b}$ B1 $(15 \mu \mathrm{mol})$, $2 \mathbf{H ~}(30 \mu \mathrm{mol})$, and acetonitrile $(500 \mu \mathrm{L}) .{ }^{c}$ Argon atmosphere. ${ }^{d} 11$ (15 $\mu \mathrm{mol}), 2 \mathrm{H}(60 \mu \mathrm{mol})$, and acetonitrile $(500 \mu \mathrm{L}) .{ }^{e}$ Mol of catalyst per mol of the limiting reagent $\left(\mathbf{1 A 1}, \mathbf{B 1}\right.$ or 11). ${ }^{f}$ The half-life time $t_{1 / 2}$ was graphically determined from the kinetics curve of dithioacetal 1A2, thioester B2 or disulfide 12 (monitored by HPLC-UV $\mathrm{Abs}_{250}$ ). ${ }^{g}$ N.R.: no product was detected. 
of this organotin compound showed a $t_{1 / 2}$ value of $25 \mathrm{~min}$ (Table 2 , entry 12, Fig. S12 $\dagger$ ). When a sub-stoichiometric amount of butylstannonic acid was used, the initial exchange was slower (Table 2, entry 13), and the product formation reached a plateau at a concentration lower than the expected value $(\cong 37 \%$, Fig. S13†). At this point, the addition of a second thiol (1-pentanethiol) did not introduce changes in the product distribution, suggesting that the catalyst was not active (Fig. S14a $\dagger$ ). In parallel, the addition of more catalyst re-started the exchange and, finally, the mixture reached the expected equilibrium composition (Fig. S14b and $\mathrm{c} \dagger$ ). Although the thioester exchange was slower with butylstannonic acid $\left(t_{1 / 2}=25 \mathrm{~min}\right)$ than with triethylamine $\left(t_{1 / 2}=0.3 \mathrm{~min}\right)$, the collateral oxidation of thiol $2 \mathbf{H}$ was also slower (Fig. $\mathrm{S} 15 \dagger$ ).

In the presence of butylstannonic acid, thioester exchange could proceed via an exchange/insertion mechanism similar to that proposed for organotin(Iv) catalyzed transesterification reactions. ${ }^{64}$ Although an efficient exchange can be achieved under base catalysis, alternative conditions for this reversible reaction may be useful for the preparation of base-labile dynamic systems. In that context, the transthioesterification process observed in the presence of butylstannonic acid may represent an interesting starting point for further studies.

Disulfide exchange is one of the most popular reactions in dynamic combinatorial chemistry. ${ }^{65}$ In aqueous media it is active at nearly neutral $\mathrm{pH}$ values, but in organic solvents a base is usually required. In our model, disulfide exchange was achieved in acetonitrile without a catalyst (Table 2, entry 14, Fig. S16 ${ }^{\dagger}$ ). In this solvent, TEA and butylstannonic acid accelerated the exchange (Table 2, entries 15-16, Fig. S17 and S18 $\dagger$ ), whereas TFA inactivated it (Table 2 , entry 17, Fig. S19†).

\section{Double-level systems based on simultaneous and communicating reversible reactions}

In order to study the interplay between different reactions, two binary combinations of simultaneous and communicating reactions were studied, disulfide-thioester and disulfidedithioacetal exchanges, and the kinetic traces of isolated versus networked reactions were compared.

Simultaneous disulfide and thioester exchanges. A simple two-level communicating system was prepared when thiol $2 \mathbf{H}$ (60 $\mathrm{mM})$ was added to an acetonitrile solution containing disulfide 11 (30 mM), thioester B1 (30 mM) and TEA (30 mM) under an argon atmosphere. Both disulfide $\mathbf{2 2}$ and thioester B2 reached a constant concentration after 2 min of reaction (Fig. 4, see full composition in Fig. S20 †). A comparison of the kinetic traces of these reactions showed some differences when they were individually (isolated) or simultaneously (networked) carried out.

Since the initial concentration of thiol $2 \mathbf{H}$ (total concentration of building block 2 in the system) was the same for isolated and networked experiments, the final proportion of disulfide and thioester products containing building block 2 in the networked reactions is lower than that in the isolated reactions (Fig. 4). As expected, this observation is independent of the

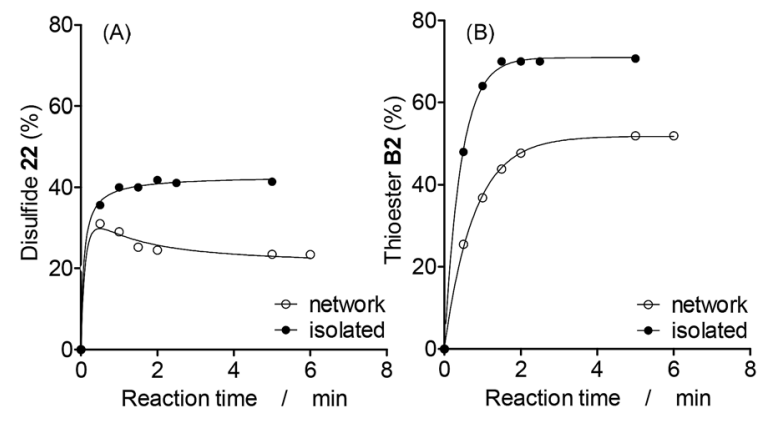

Fig. 4 Kinetics profile of the networked and isolated (A) disulfide and (B) thioester reactions. Mixture formed upon addition of thiol $2 \mathrm{H}(60$ $\mathrm{mM})$ in a solution containing TEA (30 mM), disulfide $11(30 \mathrm{mM})$ and/or thioester B1 (30 mM) under an argon atmosphere. Solvent: acetonitrile (total volume: $500 \mu \mathrm{L}$ ), r.t. The amount of 22 and $\mathrm{B} 2$ at the base of the HPLC peak areas $\left(\mathrm{Abs}_{250}\right)$ was calculated as follows: $22(\%)=22 \times 100 /$ $(11+12+22)$ and B2 $(\%)=B 2 \times 100 /(B 1+B 2)$.

catalyst used as shown by similar results observed with an alternative catalyst (Fig. S21†).

A more subtle networking effect was observed when the formation kinetics of the networked and isolated disulfide $\mathbf{2 2}$ were compared more closely. In the absence of thioesters, the amount of 22 increased until it became constant after 1 min of reaction (Fig. 4A, isolated). In the presence of exchanging thioesters, the amount of disulfide 22 reached a maximum value of $30 \%$ after $0.5 \mathrm{~min}$, and then it decreased to reach a constant amount of $24 \%$ after 2 min of reaction (Fig. $4 \mathrm{~A}$, network). Since disulfides exchange faster than thioesters, 22 is a kinetically preferred product and initially reached a maximum concentration that is higher than its concentration at equilibrium.

Simultaneous disulfide and dithioacetal exchanges. During the series of experiments shown in Table 1, a slow dithioacetal exchange in the presence of tin(II) chloride was observed (entry 10). In an attempt to accelerate the exchange, the mixture was heated at $100{ }^{\circ} \mathrm{C}$ under an argon atmosphere, and both dithioacetal and disulfide exchanges were observed to be active. A comparison of the kinetic traces obtained for the two individual (isolated) or simultaneous (networked) reactions showed similar differences to those described for thioester and disulfide exchanges.

The final proportion of product $\mathbf{2 2}$ or $\mathbf{2 A 2}$ was lower in the networked than in the isolated reactions (Fig. 5, see full composition in Fig. S22 $\dagger$ ). Under these conditions, dithioacetals exchange faster than disulfides (Fig. 5, isolated). The formation of dithioacetal $\mathbf{2 A 2}$ was kinetically favored and reached a transient maximum concentration of $23 \%$ after $0.7 \mathrm{~h}$ of reaction (Fig. 5B, network). Then, a decrease in $2 \mathrm{~A} 2$ concentration was observed to reach the final constant concentration of $20 \%$ after $2 \mathrm{~h}$.

Similar kinetic traces were observed by Lehn et al. in dynamic systems where different nitrogen nucleophiles, such as amines, alkoxy-amines and hydrazides, were competed by one aldehyde. ${ }^{32}$ As in our examples, a kinetic product is formed first; its concentration reaches a maximum and then decreases. However, the equilibrium was never reached in those systems. 

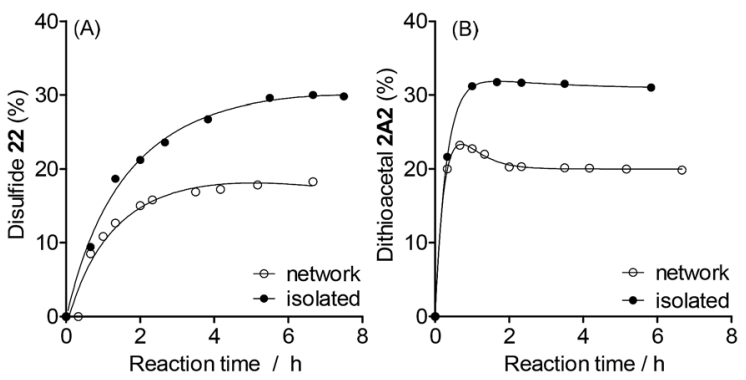

Fig. 5 Kinetics profile of the (A) disulfide and (B) dithioacetal layer in the mixture formed from dithioacetal $1 \mathrm{~A} 1(30 \mathrm{mM})$, disulfide 11 (30 $\mathrm{mM})$, thiol $2 \mathrm{H}(60 \mathrm{mM})$, and $\mathrm{SnCl}_{2}(1.5 \mathrm{mM})$. Solvent: acetonitrile (total volume: $500 \mu \mathrm{L}$ ). Temperature: $100{ }^{\circ} \mathrm{C}$ under an argon atmosphere. The amount of 22 and $2 \mathrm{~A} 2$ at the base of the HPLC peak area data $\left(\right.$ Abs $\left._{250}\right)$ was calculated as follows: $22(\%)=22 \times 100 /(11+12+22)$ and $2 \mathrm{~A} 2(\%)=2 \mathrm{~A} 2 \times 100 /(1 \mathrm{~A} 1+1 \mathrm{~A} 2+2 \mathrm{~A} 2)$.

In the future, it would be interesting to study this behaviour further by pushing the system towards more stringent competition for starting materials (changing relative building blocks' concentration).

\section{Triple-level systems based on sequential/simultaneous and communicating reactions: the emergence of multilayer networks}

The access to reaction conditions that activate dithioacetal, disulfide and/or thioester exchanges could be a good starting point to build multilayer networks. To this end, the same starting mixture of 1A1, B1, 11 and $\mathbf{2 H}$ was used to generate multilevel systems which differ in the number and order of reactions along the sequence.

The first sequence started with an acetonitrile solution containing disulfide 11 (30 mM), thioester B1 (30 mM), and dithioacetal $1 \mathrm{A1}$ (30 mM) (Fig. 6A). Initially, disulfide exchange was selectively activated by addition of thiol $2 \mathbf{H}(60 \mathrm{mM})$. Under these conditions, thioester and dithioacetal exchanges were inactive (Fig. 6B).

Next, disulfide exchange was stopped and dithioacetal exchange was activated by adding $\mathrm{H}_{2} \mathrm{SO}_{4}$. In this step, a net transference of building block 2 from the thiol pool to the dithioacetal pool was observed generating 1A2 and 2A2 (Fig. 6C). The associated transference of building block 1 in the opposite direction was also noticeable: the $\mathbf{1 H} / \mathbf{2 H}$ peak area proportion increased from 1 (Fig. 6B) to 2 (Fig. 6C). Although at this point disulfides and thioesters stayed disconnected from the network (Fig. 7, 2nd layer of sequence I), the information imprinted in the relative concentration of the free thiols was later transferred to both pools when TEA was added. In the third layer, disulfides and thioesters containing building block 1 were favored. The peak areas of disulfides 11 (42\%) and 12 (46\%) were higher than that of 22 (12\%) (Fig. 6D), an opposite situation when compared to the relative concentration of these disulfides when only disulfide exchange was active (Fig. 6B).

Computer simulations can be useful to analyze the behavior of dynamic combinatorial libraries. ${ }^{66-70}$ Simulations were carried out in order to predict the composition of the

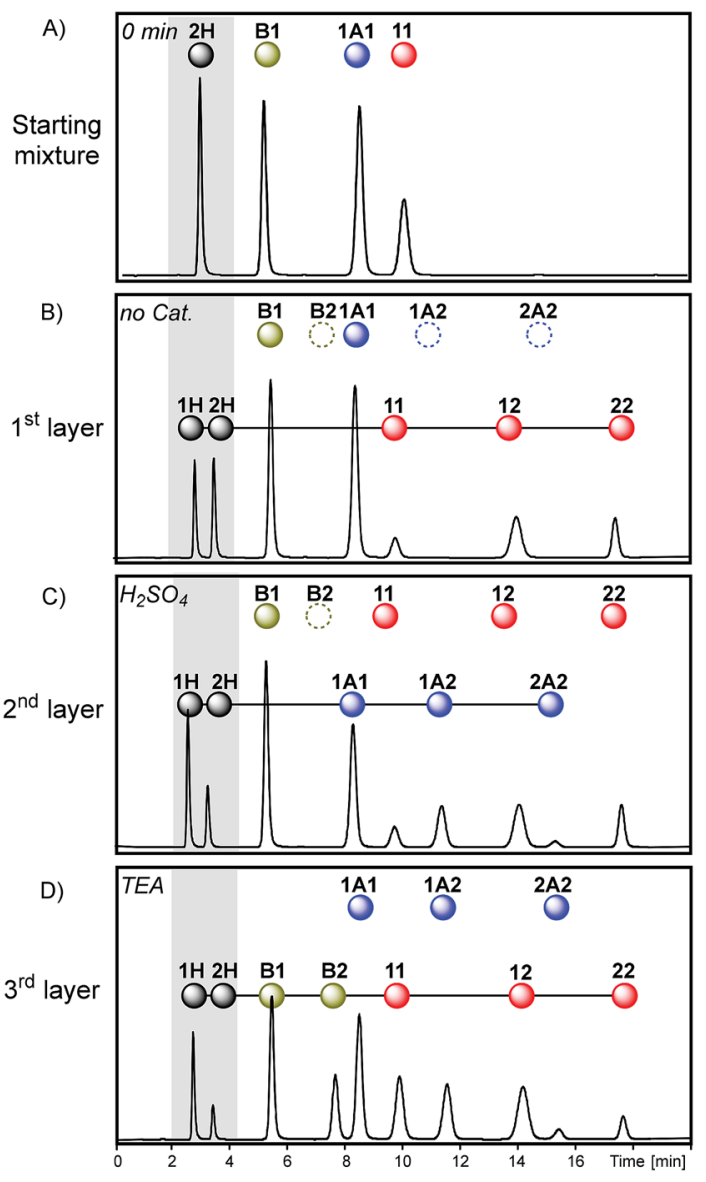

Fig. 6 Chromatograms $\left(\mathrm{Abs}_{250}\right)$ obtained in each step of sequence I. Starting mixture $(A)$, after the equilibrium was reached in the absence of any catalyst ( $\mathrm{B}$, first layer), in the presence of $\mathrm{H}_{2} \mathrm{SO}_{4}$ ( $\mathrm{C}$, second layer) and later in the presence of an excess of TEA ( $D$, third layer).

equilibrated libraries constructed with sequential reactions. The experimentally observed equilibrium compositions after each activation step (Fig. 6) were close to those observed in a simulated multilevel system with isoenergetic components (Fig. S23†). This suggests that the library composition was not influenced by non-covalent interactions between library members and/or catalysts. Therefore, the progress of the composition over time (first, second, and third layers of the network) was defined by the covalent network and the relative stability of the components.

Analysis of product distributions along sequence I illustrates how the information stored at the thiol connector level can be transferred between layers. Although dithioacetals and disulfides (or thioesters) were never connected to the network at the same time (Fig. 7, sequence I), the final distribution of disulfides and thioesters was affected by the proportion of dithioacetals in the previous layer (due to its push-pull effect on thiols).

It is expected that the relative amount of free thiols $\mathbf{1 H}$ and 2H will change during the sequential process in different manners when different sequences are generated. Since these thiols connect different layers, the relative proportion given by the equilibration in one layer will probably affect the equilibrium composition in the subsequent level. 


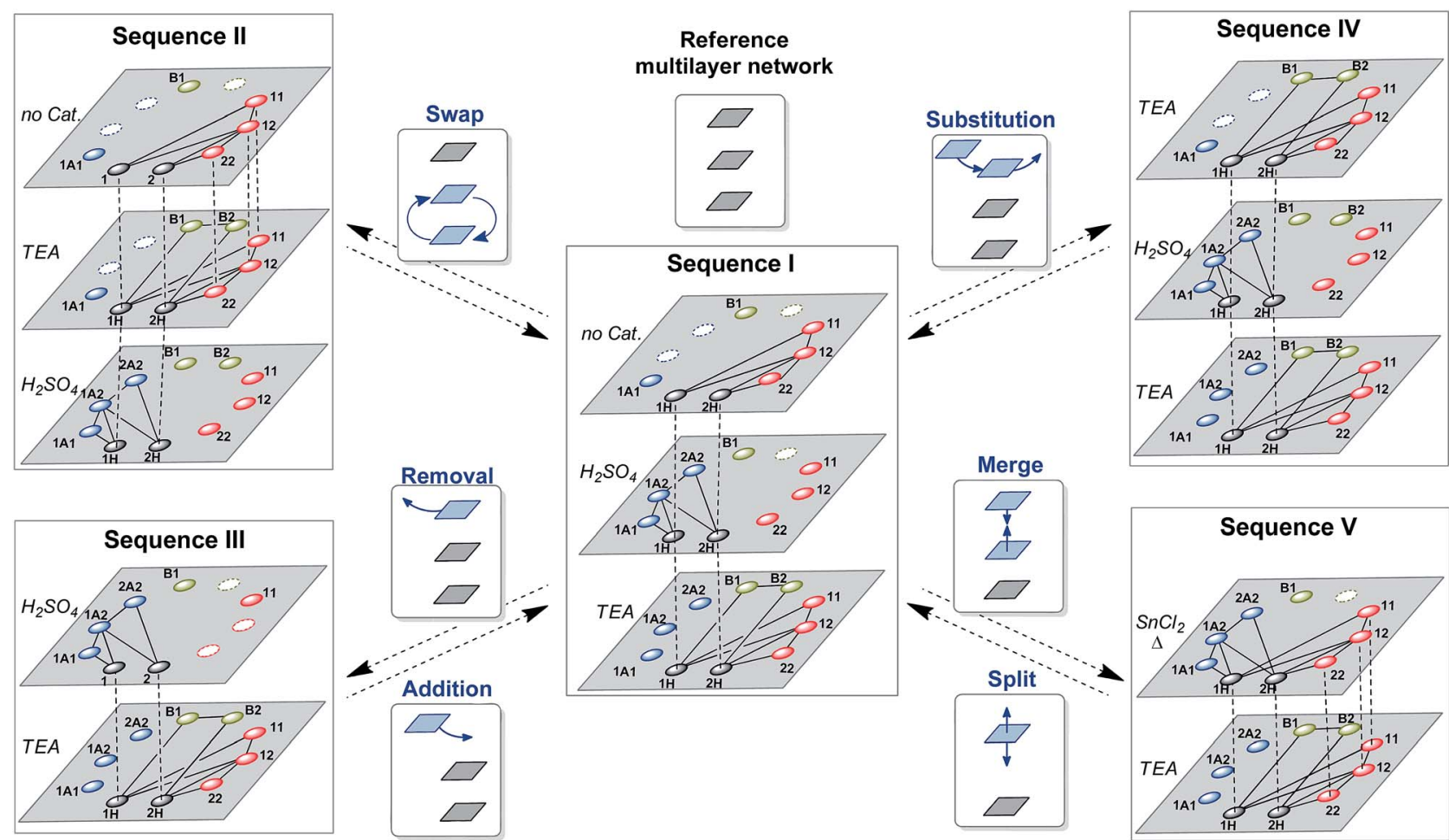

Fig. 7 Engineering multilayer chemical networks in dynamic multilevel systems. The introduction of changes into a reference multilayer network gives rise to a variety of networks upon application of operations based on swapping, removal, substitution or merging of layers.

\section{Network engineering: a new level of design in synthetic chemical systems}

Multilevel dynamic systems are usually designed taking in consideration chemical properties such as compatibility between the reversible reactions applied, the effect of the molecular structure on the reactivity, the timing of the reactions, etc. The emergence of multilayer networks gives rise to other design aspects such as the layer order and number. The impact of such features on the final composition was analyzed through some operations at the network level, including layer swapping, removal, substitution and merging.

The order in which the layers of the multilayer network are stacked can be altered by changing the activation order of the reactions along the sequence. For instance, the second and third layers of sequence I (Fig. 7) can be swapped by inverting the order of addition of $\mathrm{H}_{2} \mathrm{SO}_{4}$ and TEA to produce sequence II (Fig. 7). In sequence II disulfides were initially exchanged as in the first layer of sequence I, and then thioesters were connected to the network through the addition of TEA (2nd layer of sequence II) and, finally, $\mathrm{H}_{2} \mathrm{SO}_{4}$ was added to stop the thioester/ disulfide exchange and to activate dithioacetal exchange (3rd layer of sequence II). Therefore, once disulfides and thiols were equilibrated (Fig. 8A), thioester exchange was activated with TEA, keeping disulfide exchange active. The connection of thioesters to the network produced the net transference of building block 1 from B1 to the thiol pool and, consequently, to the disulfide pool (Fig. 8B). The proportion of peak areas of disulfides 11/22 increased from 0.4 in the first layer (Fig. 8A) to 1.0 in the second layer (Fig. 8B). In parallel, a concomitant transference of building block 2 from disulfide 22 and thiol $2 \mathbf{H}$ to form thioester B2 was observed (Fig. 8B). Next, disulfide and thioester exchanges were stopped and dithioacetal exchange

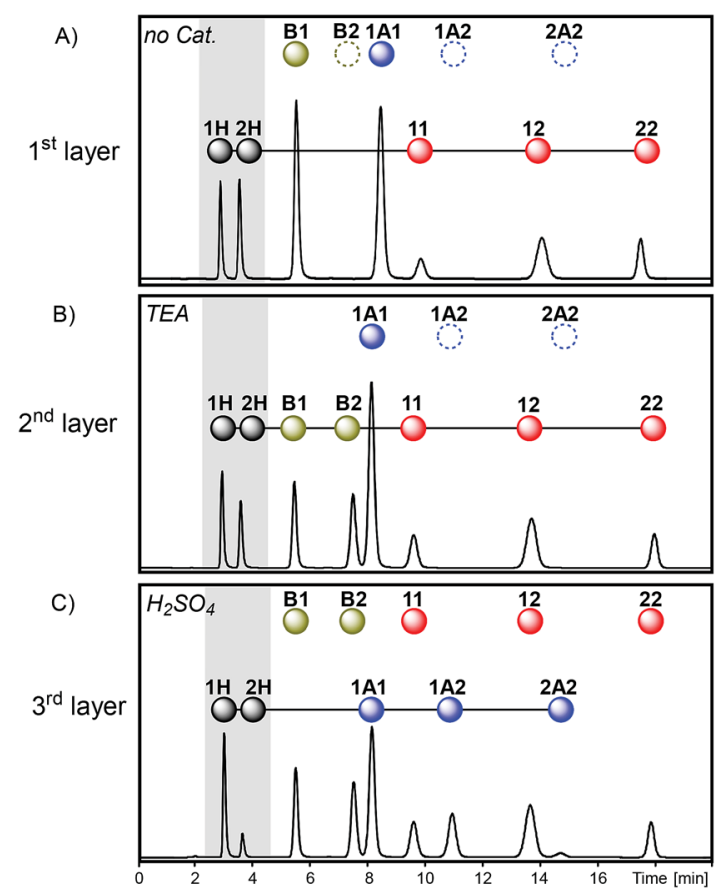

Fig. 8 Chromatograms $\left(\mathrm{Abs}_{250}\right)$ obtained in each step of sequence II after the equilibrium was reached in the absence of any catalyst ( $A$, first layer), in the presence of TEA ( $B$, second layer) and later in the presence of an excess of $\mathrm{H}_{2} \mathrm{SO}_{4}$ (C, third layer). 
was activated by adding $\mathrm{H}_{2} \mathrm{SO}_{4}$. In this step, a net transference of building block 2 from the thiol pool to the dithioacetal pool was observed, generating $\mathbf{1 A 2}$ and $2 \mathrm{~A} 2$ (Fig. 8C). The associated transference of building block 1 in the opposite direction (from the dithioacetal pool to the thiol pool) was also noticeable, and the $\mathbf{1 H} / \mathbf{2 H}$ proportion increased from 1.3 in the second layer to 4.8 in the third layer (Fig. 8B and C, respectively).

It is worth mentioning, though, that the networks of the last layer of sequences I and II are different (Fig. 7) and this could affect the product distribution. In order to evaluate the effect of the history on the final product distributions of multilayer dynamic systems wherein the last layer is always the same network, three operations that do not involve the third layer of sequence I were studied: layer removal, layer substitution and layer merging (Fig. 7, sequences III-IV).

Thus, sequence III was obtained through the removal of the first layer of sequence I (Fig. 7). In this case, the initial dithioacetal exchange led to an equilibrium composition dominated by products that contain building block 2: the proportion of peak areas $1 \mathrm{H} / 2 \mathrm{H}$ was 0.7 while the proportion of $1 \mathrm{A1} / 2 \mathrm{~A} 2$ was 0.5 (Fig. 9A). This is because building block $\mathbf{1}$ is mostly trapped in the form of thioester $\mathbf{B 1}$ and disulfide $\mathbf{1 1}$ so it cannot be exchanged under acid catalysis. Next, the dithioacetal level was blocked while the disulfide-thioester level was activated. At this stage, a net transference of building block 2 was observed from the thiol pool towards the thioester and disulfide pools, to produce B2, 12 and 22 at the expense of $\mathbf{2 H}$ (the $\mathbf{1 H} / \mathbf{2 H}$ peak area proportion increases from 0.7 to 4.0 , Fig. 9A versus 9B).

A comparison of the final compositions for sequences I and III illustrates the influence of history. These two mixtures have the same components and the same network topology (see the last layers of sequences I and III in Fig. 7). However, the relative product concentrations at the equilibrium of these two dynamic mixtures were different. The final layer of sequence III had a proportion of dithioacetals $1 \mathrm{A1} / 2 \mathrm{~A} 2=0.5$ (Fig. 9B), significantly lower than the value of 9.5 observed for sequence I (Fig. 6D).

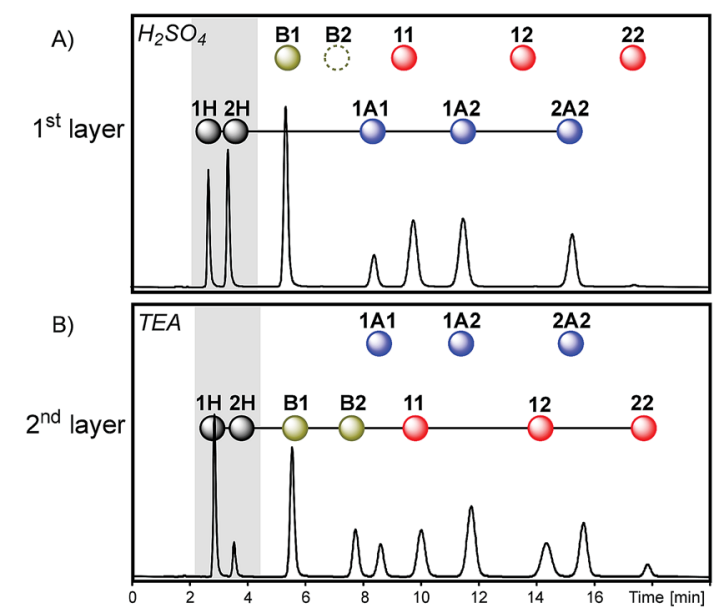

Fig. 9 Chromatograms ( $\mathrm{Abs}_{250}$ ) obtained in each step of sequence III after the equilibrium was reached in the presence of $\mathrm{H}_{2} \mathrm{SO}_{4}$ ( $\mathrm{A}$, first layer) and later in the presence of an excess of TEA (B, second layer).
In sequence I, dithioacetal exchange was activated after equilibration of disulfide exchange and, as a consequence, part of building block 2 was stored in the disulfide pool and was unavailable for exchange in the acidic medium. In sequence III, since the first layer was removed, dithioacetal exchange was the first active reaction and a higher amount of 2 could be processed by the dithioacetal pool.

If the first layer of sequence I remains the same, and the second layer is removed, again, a different final composition is achieved. Since in the reference sequence I, dithioacetal exchange was active only in the second layer, removal of this layer led to a final distribution wherein 1A1 was the only dithioacetal present (Fig. 8B).$^{71}$ Because building block 2 was never incorporated into dithioacetal products along this sequence $\mathrm{II}_{\text {short }}$, the final relative concentrations B1/B2 and 11/ 22 were lower than those in sequence I.

An alternative way of affecting the system history is through the substitution of the first layer of sequence I by a different layer to produce sequence IV (Fig. 7). Initially, disulfide and thioester exchanges were activated in the presence of TEA, and then sulfuric acid was added to stop both exchanges and to activate the exchange of dithioacetal products and, finally, a new addition of TEA (in excess) activated disulfide and thioester exchanges and simultaneously deactivated dithioacetal exchange (Fig. 10).

From the network-wiring point of view, the only difference between sequences I and IV is the connection of thioester

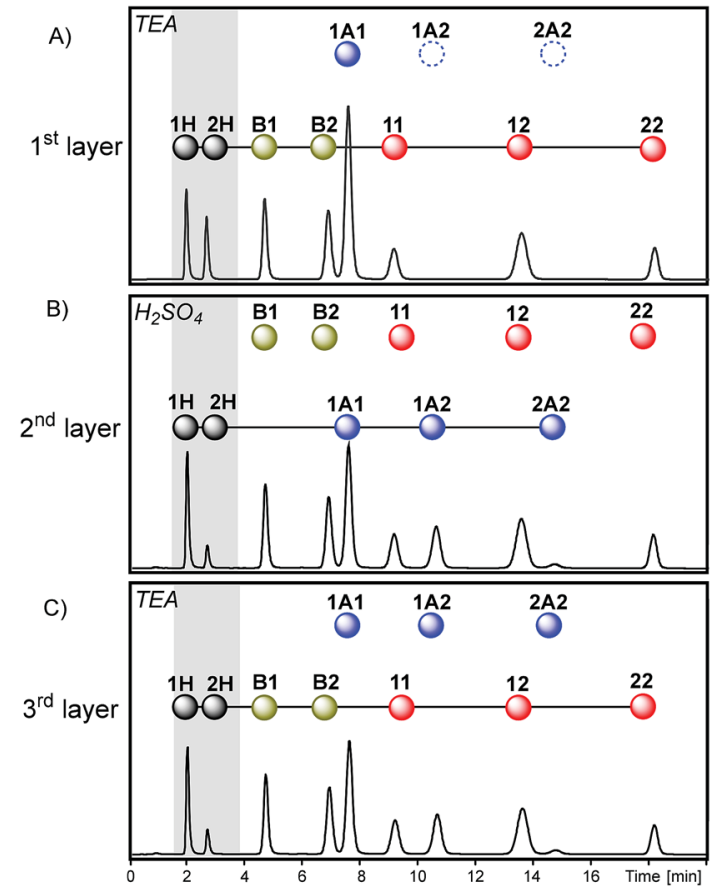

Fig. 10 Chromatograms ( $\mathrm{Abs}_{250}$ ) obtained in each step of sequence IV after the equilibrium was reached in the presence of TEA ( $A$, first layer), in the presence of an excess of $\mathrm{H}_{2} \mathrm{SO}_{4}$ ( $\mathrm{B}$, second layer) and later in the presence of an excess of TEA ( $C$, third layer). The sequence started from a mixture containing dithioacetal $1 \mathrm{~A} 1(30 \mathrm{mM})$, thioester B1 (30 $\mathrm{mM})$, disulfide $11(30 \mathrm{mM})$ and $2 \mathrm{H}(60 \mathrm{mM})$. 
exchange to the first layer of sequence IV. This difference was translated into significant differences in the relative concentration of the thioesters $\mathbf{B} 1$ and $\mathbf{B} 2$ at the end of the sequence (the proportions of peak areas B1/B2 were 1.7 and 0.9 in sequences I and IV respectively, Fig. 6D and 10C). The lower relative concentration of $\mathbf{B} 2$ is due to the fact that in sequence I thioester exchange was activated only after some building block 2 had been trapped as dithioacetals, whereas in sequence IV the thioester exchange was activated, for the first time, before dithioacetal exchange.

Finally, a more subtle way to change the system history was attempted by merging the first two layers. With this in mind, the reactions involved in the first and second layers of sequence I were simultaneously activated leading to sequence V (Fig. 7).

Simultaneous dithioacetal and disulfide exchanges $\left(\mathrm{SnCl}_{2} /\right.$ heat) provided a composition where compounds containing building block 1 were present in a higher amount, as indicated by the relative peak areas: $\mathbf{1 H}>\mathbf{2 H}, \mathbf{1 1}>\mathbf{2 2}$ and $1 \mathrm{A1}>\mathbf{2 A 2}$ (Fig. 11A). Further inactivation of dithioacetal exchange at the time that thioester exchange was activated (along with disulfide exchange) led to a net transference of building block 2 from the disulfide pool to the thioester pool. The most evident change was the appearance of the thioester B2 signal, which is mainly formed with the building block coming from disulfide 22 (Fig. 11B). As previously observed for sequence I, numerical simulations of the equilibrium composition after each activation step of sequences II-V lead to product distributions that resemble those observed experimentally (Fig. S24-S27†). The simulations indicate that the catalysts used did not affect the relative stability of the library members, i.e. the product distribution. Therefore, the differences observed in the final composition of sequences I-IV are defined only by the activation order of the different reversible reactions.

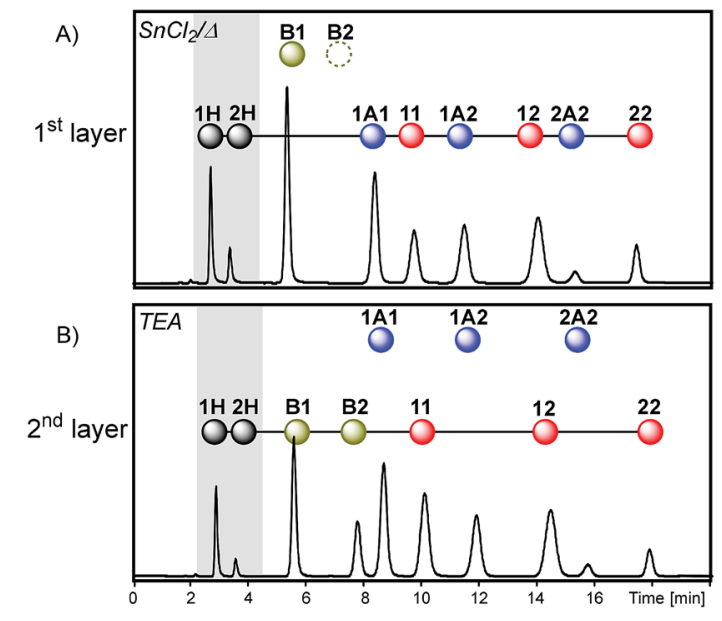

Fig. 11 Chromatograms $\left(\mathrm{Abs}_{250}\right.$ ) obtained in each step of sequence $\mathrm{V}$ starting from a mixture containing dithioacetal $1 \mathrm{~A} 1(30 \mathrm{mM})$, thioester $\mathrm{B} 1(30 \mathrm{mM})$, disulfide $11(30 \mathrm{mM}), 2 \mathrm{H}(60 \mathrm{mM})$ and $\mathrm{SnCl}_{2}(1.5 \mathrm{mM})$. Sequential addition of catalysts/conditions is as follows: (A) $960 \mathrm{~min}$ after $100^{\circ} \mathrm{C}$ heating, Ar atmosphere and (B) 10 min after the addition of TEA (300 mM), Ar atmosphere. Solvent: acetonitrile (total volume: 500 $\mu \mathrm{L})$.
A comparison of the final product distribution of sequences $I-V$ illustrates how some changes in a layer, early in the sequence, can be read in the last layer (Fig. 12). In this extremely simple multilayer system, the final product distribution depended on the order in which the different reversible reactions had been previously activated/deactivated. Communication between the reversible reactions used allowed spreading of building blocks 1 and 2 over the different pools of compounds (thioesters, disulfides, dithioacetals and thiols). The order of activation/deactivation of the different reactions influenced the proportion in which these building blocks accumulated within each pool.

In the model used, the total amount of $\mathbf{1}$ is 2.5 times the amount of 2 , which introduces a bias towards products containing the former building block. This preference is observed in the final product distribution of sequence $\mathrm{I}$ : $[\mathbf{B 1}]>[\mathbf{B 2}],[\mathbf{1 1}]>$ [22] and [1A1] > [2A2] (Fig. 12). Some of the operations with the layers of sequence I inverted this preference within the different compound pools. Removal of the second layer (to produce sequence $\mathrm{II}_{\text {short }}$ ) or substitution of the first layer (to produce sequence IV) leads to final compositions wherein $[\mathbf{B 1}]<[\mathbf{B 2}]$ and $[11] \leq[22]$ (Fig. 12). Interestingly, in the first case (sequence $\mathrm{II}_{\text {short }}$ ) the product preference within the disulfide and thioester pools was reverted when a dithioacetal-related layer was removed. In the second case (sequence IV), the product preferences within the disulfide and the thioester pools were reverted through the rewiring of a layer where these reactions are involved, i.e. the connection of the thioester nodes to the network (compare the 1st layer of sequence I $v s$. the 1st layer of sequence IV in Fig. 7).

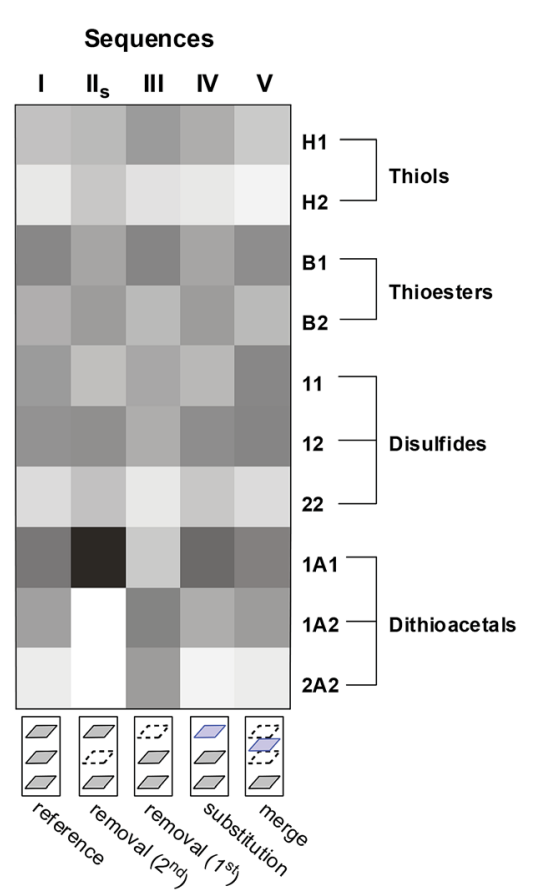

Fig. 12 Heat map plot showing the final abundances of library members for the different sequences. Shade represents the peak area normalized to the total peak area. 
Likewise, removal of the first layer (sequence III) reverted the preference at the dithioacetal level leading to a final composition where $[\mathbf{1 A 1}]<[\mathbf{2 A 2}]$ (Fig. 12). Again, in this case the product preference within the dithioacetal pool was reverted by the removal of a layer that involves only the exchange of disulfides.

\section{Conclusions}

In systems with a single reversible reaction, composition changes can be studied under out-of-equilibrium conditions. Once at equilibrium, the situation is rather different. By definition, thermodynamic equilibrium is a state of maximum entropy, so no information about the history of the system can be stored in it.

By judicious choice of reactions and conditions, different single-reaction systems can be coupled to produce multilayer networks that include strings of reversible reactions.

Two interesting consequences arise in systems where sequential reversible reactions are communicated through common nodes. First, the activation/deactivation order of the reactions gives the chemical pathway a direction in the time function, ${ }^{72}$ which configures the history of the system.

Second, given that some compounds (connectors) act as starting materials and as products of different reactions, their concentration can affect the composition of different exchange pools. Thus, thermodynamic systems with sequential reactions can keep track of previous equilibration events in their history. This attribute can be particularly interesting if we consider the intrinsic adaptability of each layer (granted by the use of reversible chemistry). Environmental changes in early layers could be imprinted in final product distributions even if they no longer exist at the time the sequence has finished.

Altogether, these features give rise to a new design level that, in our opinion, deserves further exploration.

Adaptability is a key feature of dynamic covalent chemistry. Such a property has led to the discovery of new functional materials, ${ }^{3-7}$ drugs $^{8-10}$ and catalysts. ${ }^{73}$ Multilayer chemical network engineering can prove useful in the functional screening of dynamic combinatorial libraries (DCLs). By judicious choice of reaction conditions, a library can be biased toward different layers giving access to the modular exploration of different regions of chemical space and connectivity.

DCL adaptation has also been used to sense the presence of analytes. ${ }^{\mathbf{1 3 , 1 4}}$ In multilayer systems, the introduction of the time dimension can be useful to sense analytes added at different times. Such information could be read in the final composition.

Systems chemistry aims to develop molecular systems of interacting and/or interconverting molecules that show emergent properties. ${ }^{2,23}$ The preparation of complex reaction networks constitutes an integral part of systems chemistry. ${ }^{74-76}$ In this context, dynamic systems that can respond to environmental changes (light, acidity, metals, etc.) by switching their type of reversible chemistry and connectivity can represent an exciting study case for emergent behaviour.

\section{Conflicts of interest}

There are no conflicts to declare.

\section{Acknowledgements}

The project was funded by the Fondo para la Investigación Científica y Tecnológica (FONCYT) (PICT2015-3574), Consejo Nacional de Investigaciones Científicas y Técnicas (CONICET), and Universidad Nacional de Rosario. M. M.-A. acknowledges CONICET for a postdoctoral fellowship. A. G. O. and R. L. E. F. are CONICET researchers.

\section{Notes and references}

1 J.-M. Lehn, Angew. Chem., Int. Ed., 2015, 54, 3276-3289.

2 J.-M. Lehn, Chem. Soc. Rev., 2007, 36, 151-160.

3 Y. Zhang and M. Barboiu, Chem. Rev., 2016, 116, 809-834.

4 Z. Wei, J. H. Yang, J. Zhou, F. Xu, M. Zrínyi, P. H. Dussault, Y. Osada and Y. M. Chen, Chem. Soc. Rev., 2014, 43, 81148131.

5 E. Moulin, G. Cormos and N. Giuseppone, Chem. Soc. Rev., 2012, 41, 1031-1049.

6 J.-M. Lehn, Prog. Polym. Sci., 2005, 30, 814-831.

7 T. Maeda, H. Otsuka and A. Takahara, in Dynamic Combinatorial Chemistry, John Wiley \& Sons, Inc., Hoboken, NJ, USA, 2009, pp. 229-260.

8 Dynamic combinatorial chemistry: in drug discovery, bioorganic chemistry, and materials science, ed. B. L. Miller, Wiley, 2009.

9 S. Otto, R. L. E. Furlan and J. K. M. Sanders, Drug Discovery Today, 2002, 7, 117-125.

10 O. Ramström and J.-M. Lehn, Nat. Rev. Drug Discovery, 2002, 1, 26-36.

11 S. Ulrich, Acc. Chem. Res., 2019, 52, 510-519.

12 E. Bartolami, C. Bouillon, P. Dumy and S. Ulrich, Chem. Commun., 2016, 52, 4257-4273.

13 A. Herrmann, Chem.-Eur. J., 2012, 18, 8568-8577.

14 S. Otto and K. Severin, in Creative Chemical Sensor Systems, Springer Berlin Heidelberg, Berlin, Heidelberg, 2007, pp. 267-288.

15 Y. Liu and K. C.-F. Leung, in Dynamic Covalent Chemistry: Principles, Reactions and Applications, John Wiley \& Sons, Ltd, Chichester, UK, 2017, pp. 287-319.

16 D. A. Leigh, U. Lewandowska, B. Lewandowski and M. R. Wilson, Molecular Machines and Motors, in Topics in Current Chemistry, ed. A. Credi, S. Silvi and M. Venturi, 2014, vol. 354, pp. 111-138.

17 M. von Delius and D. A. Leigh, Chem. Soc. Rev., 2011, 40, 3656-3676.

18 J. Li, P. Nowak and S. Otto, J. Am. Chem. Soc., 2013, 135, 9222-9239.

19 R. A. R. Hunt and S. Otto, Chem. Commun., 2011, 47, 847858.

20 A. G. Orrillo, A. M. Escalante, M. Martinez-Amezaga, I. Cabezudo and R. L. E. Furlan, Chem.-Eur. J., 2019, 25, 1118-1127.

21 S. Otto, Acc. Chem. Res., 2012, 45, 2200-2210.

22 G. Ashkenasy, T. M. Hermans, S. Otto and A. F. Taylor, Chem. Soc. Rev., 2017, 46, 2543-2554.

23 O. Š. Miljanić, Chem, 2017, 2, 502-524. 
24 A. S. Y. Wong and W. T. S. Huck, Beilstein J. Org. Chem., 2017, 13, 1486-1497.

25 A. Wilson, G. Gasparini and S. Matile, Chem. Soc. Rev., 2014, 43, 1948-1962.

26 H. M. Seifert, K. Ramirez Trejo and E. V. Anslyn, J. Am. Chem. Soc., 2016, 138, 10916-10924.

27 S. Lascano, K.-D. Zhang, R. Wehlauch, K. Gademann, N. Sakai and S. Matile, Chem. Sci., 2016, 7, 4720-4724.

28 A. M. Escalante, A. G. Orrillo and R. L. E. Furlan, J. Comb. Chem., 2010, 12, 410-413.

29 L. Rocard, D. Wragg, S. A. Jobbins, L. Luciani, J. Wouters, S. Leoni and D. Bonifazi, Chem.-Eur. J., 2018, 24, 1613616148.

30 R. C. Lirag and O. Š. Miljanić, Chem. Commun., 2014, 50, 9401-9404.

31 J. Leclaire, L. Vial, S. Otto and J. K. M. Sanders, Chem. Commun., 2005, 1959-1961.

32 S. Kulchat, M. N. Chaur and J.-M. Lehn, Chem.-Eur. J., 2017, 23, 11108-11118.

33 J. F. Reuther, S. D. Dahlhauser and E. V. Anslyn, Angew. Chem., Int. Ed., 2019, 58, 74-85.

34 B. M. Matysiak, P. Nowak, I. Cvrtila, C. G. Pappas, B. Liu, D. Komáromy and S. Otto, J. Am. Chem. Soc., 2017, 139, 6744-6751.

35 Y. Hai, H. Zou, H. Ye and L. You, J. Org. Chem., 2018, 83, 9858-9869.

36 A. G. Orrillo, A. La-Venia, A. M. Escalante and R. L. E. Furlan, Chem.-Eur. J., 2018, 24, 3141-3146.

37 B. T. Worrell, S. Mavila, C. Wang, T. M. Kontour, C.-H. Lim, M. K. McBride, C. B. Musgrave, R. Shoemaker and C. N. Bowman, Polym. Chem., 2018, 9, 4523-4534.

38 S. Otto, R. L. E. Furlan and J. K. M. Sanders, J. Am. Chem. Soc., 2000, 122, 12063-12064.

39 S. J. Rowan, S. J. Cantrill, G. R. Cousins, J. K. Sanders and J. F. Stoddart, Angew. Chem., Int. Ed. Engl., 2002, 41, 898-952.

40 A. G. Orrillo, A. M. Escalante and R. L. E. Furlan, Chem.-Eur. J., 2016, 22, 6746-6749.

41 A. G. Orrillo, A. M. Escalante and R. L. E. Furlan, Org. Lett., 2017, 19, 1446-1449.

42 Y.-C. Wu and J. Zhu, J. Org. Chem., 2008, 73, 9522-9524.

43 S. K. De, Tetrahedron Lett., 2004, 45, 2339-2341.

44 B. Tamami and K. P. Borujeny, Synth. Commun., 2003, 33, 4253-4258.

45 H. K. Patney, Tetrahedron Lett., 1991, 32, 2259-2260.

46 R. V. Anand, P. Saravanan and V. K. Singh, Synlett, 1999, 1999, 415-416.

47 B. Cazes and S. Julia, Tetrahedron Lett., 1978, 19, 4065-4068. 48 P. Stütz and P. A. Stadler, Org. Synth., 1977, 56, 8.

49 B. M. Lamb and C. F. Barbas III, Chem. Commun., 2015, 51, 3196-3199.

50 K. Du, S. Wang, R. S. Basha and C. Lee, Adv. Synth. Catal., 2019, 361, 1597-1605.

51 O. A. Mascaretti and R. L. E. Furlan, Aldrichimica Acta, 1997, 30, 55-68.
52 L. R. Sutton, W. A. Donaubauer, F. Hampel and A. Hirsch, Chem. Commun., 2004, 1758-1759.

53 P. A. Brady, R. P. Bonar-Law, S. J. Rowan, C. J. Suckling and J. K. M. Sanders, Chem. Commun., 1996, 319-320.

54 S. J. Rowan, P. A. Brady and J. K. M. Sanders, Angew. Chem., Int. Ed. Engl., 1996, 35, 2143-2145.

55 R. Larsson, Z. Pei and O. Ramström, Angew. Chem., Int. Ed., 2004, 43, 3716-3718.

56 R. Larsson and O. Ramström, Eur. J. Org. Chem., 2006, 2006, 285-291.

57 Y. Zhang, M. Angelin, R. Larsson, A. Albers, A. Simons and O. Ramström, Chem. Commun., 2010, 46, 8457-8459.

58 S. Ghosh, L. A. Ingerman, A. G. Frye, S. J. Lee, M. R. Gagné and M. L. Waters, Org. Lett., 2010, 12, 1860-1863.

59 Y. Ura, J. M. Beierle, L. J. Leman, L. E. Orgel and M. R. Ghadiri, Science, 2009, 325, 73-77.

60 Y. Ruff, V. Garavini and N. Giuseppone, J. Am. Chem. Soc., 2014, 136, 6333-6339.

61 E. B. Hadley and S. H. Gellman, J. Am. Chem. Soc., 2006, 128, 16444-16445.

62 S. A. Berg and B. J. Ravoo, Soft Matter, 2014, 10, 69-74.

63 C. Ghobril, K. Charoen, E. K. Rodriguez, A. Nazarian and M. W. Grinstaff, Angew. Chem., Int. Ed., 2013, 52, 1407014074.

64 M. R. Meneghetti and S. M. P. Meneghetti, Catal. Sci. Technol., 2015, 5, 765-771.

65 S. P. Black, J. K. M. Sanders and A. R. Stefankiewicz, Chem. Soc. Rev., 2014, 43, 1861-1872.

66 P. T. Corbett, S. Otto and J. K. M. Sanders, Chem.-Eur. J., 2004, 10, 3139-3143.

67 J. Atcher, A. Moure and I. Alfonso, Chem. Commun., 2013, 49, 487-489.

68 K. Severin, Chem.-Eur. J., 2004, 10, 2565-2580.

69 A. G. Orrillo and R. L. E. Furlan, J. Org. Chem., 2010, 75, 211214.

70 M. C. Misuraca, E. Moulin, Y. Ruff and N. Giuseppone, New J. Chem., 2014, 38, 3336-3349.

71 Removal of the second layer of sequence I leads to a sequence that is equivalent to the first two layers of sequence II (sequence II short).

72 O. Penrose, in Chance in physics: foundations and perspectives, ed. J. Bricmont, D. Durr, M. C. Galavotti, G. C. Ghirardi, F. Petruccione and N. Zanghi, Springer, Berlin, 2001, pp. 6182.

73 G. Gasparini, M. D. Molin and L. J. Prins, Eur. J. Org. Chem., 2010, 13, 2429-2440.

74 S. N. Semenov, L. J. Kraft, A. Ainla, M. Zhao, M. Baghbanzadeh, V. E. Campbell, K. Kang, J. M. Fox and G. M. Whitesides, Nature, 2016, 537, 656-660.

75 F. Schaufelberger and O. Ramström, J. Am. Chem. Soc., 2016, 138, 7836-7839.

76 Z. Dadon, N. Wagner, S. Alasibi, M. Samiappan, R. Mukherjee and G. Ashkenasy, Chem.-Eur. J., 2015, 21, 648-654. 